\title{
Critical behavior of a probabilistic cellular automaton describing a biological system
}

\author{
Neli R.S. Ortega ${ }^{a}$, C. Felipe de S. Pinheiro ${ }^{\text {, }}$ Tânia Tomé*, \\ J.R. Drugowich de Felício ${ }^{b}$ \\ a Instituto de Física, Universidade de São Paulo, Caixa Postal 66318, 05315-970 São Paulo, SP, Brazil \\ ${ }^{\mathrm{b}}$ Faculdade de Filosofia, Ciências e Letras, de Ribeirão Preto, Universidade de São Paulo, 14040-901 \\ Ribeirão Preto, SP, Brazil
}

Received 26 June 1997; revised 18 December 1997

\begin{abstract}
We study nonequilibrium phase transitions occurring in a probabilistic cellular automaton which describes one part of the immune system. In this model, each site can be occupied by three type of cells and the immune response under parasitic infections is described in terms of two parameters $p$ and $r$. The local rules governing the evolution of this automaton possess "up-down" symmetry similar to Ising models. Performing Monte Carlo simulations on square and cubic lattices we verify that the model displays continuous kinetic phase transitions with spontaneous symmetry breaking. We present detailed simulations and analysis of the critical behavior. Our results indicate that the model belongs to the Ising universality class, supporting the "up-down" conjecture. (c) 1998 Elsevier Science B.V. All rights reserved
\end{abstract}

\section{Introduction}

The universality in nonequilibrium phase transitions is one of the most instigating questions that have been treated in the last decades. One approach in the study of this problem is based on the construction of irreversible stochastic lattice models, that is, stochastic dynamics defined in lattices and composed of irreversible rules. These models do not have an underlying Hamiltonian and are described by the dynamics itself. Universality in these systems has been studied by approaches which consider an associated Langevin equation to the processes and by considering the features of the irreversible rules and symmetries of the lattice and order parameter. In these contexts two conjectures have been established: the "up-down" conjecture introduced by Grinstein et al. [1] and the directed percolation conjecture introduced by

\footnotetext{
* Corresponding author. Fax: +55(11)8134334; e-mail: ttome@fge.if.usp.br.
} 
Grassberger [2] and by Janssen [3] independently. The "up-down" conjecture says that if a stochastic lattice model evolves according to a dynamics that possesses "updown" symmetry and respect translational invariance then the continuous kinetic phase transition that could occur in the model is in the universality class of (reversible) Ising model. This means that for these kind of models irreversibility is irrelevant under renormalization. It contrasts to the case of models that have absorbing states for which the critical behavior belongs to the directed percolation class ("directed percolation" conjecture) where irreversibility is important in the criticality even on large scales.

Besides being of great importance in the study of universality in kinetic phase transitions it is notable that stochastic lattice models can be very appropriated to study irreversible processes occurring in systems considered in different areas of knowledge. They are specially important to mimic systems in the area of biological sciences, and we can see at least three topics that have been extensively studied: population dynamics [4-6], epidemiology [7,8] and immunology [9-14]. In fact this statistical-mechanical approach, with local rules, takes into account fluctuations which can play an important role in determining the critical behavior of these systems. So, from the point of view of nonequilibrium statistical mechanics and from the point of view of the description of different systems in biological sciences, these models are very useful due to their simplicity and capacity of explaining very intricate behavior which is not well understood yet.

Here we consider a probabilistic cellular automaton which was introduced by Tomé and Drugowich de Felício [15] to describe one part of the cellular immune system $[11,13,14,16-18]$. The automaton, which is similar to the one devised by Brass et al. $[13,14]$, was constructed to mimic the part of the immune system constituted by the $T$-helper cells. The main purpose of the model is to explain the polarization of those cells, i.e. the splitting of the $T$-helper cells between two kinds, $T_{H} 1$ or $T_{H} 2$, of mature cells, when the conditions of parasitic infections are varied. As it has been emphasized, in recent studies [16-18], the immune response will depend on which kind of $T$-helper cells will predominate on high-level infection. For example, in the case of infection with leishmania $[14,18]$ if $T_{H} 1$ cells predominate the individual will be protected, otherwise $\left(T_{H} 2\right.$ response) susceptible. It is important to note that only under a high enough level of infection the system will give different type of immune response. Under low levels of infection the number of $T_{H} 1$ cells is equal to the number of $T_{H} 2$ cells. This phenomenon is described in the model by the spontaneous symmetry breaking that takes place under a critical level of infection. In Ref. [15] numerical evidences indicated that the model can display a steady state with predominance of one type of cells. Under this condition, the model shows that the immune response is such that the system will be protected or susceptible with respect to one disease.

The present work is concerned to two questions: the universality in dynamic phase transitions and the description of $T$-helper cells response under parasitic infections. We perform Monte Carlo simulations and obtain the critical behavior of the automaton above cited (introduced in [15]). From the cumulant method [19] and surface correlation criterion [20] we construct the phase diagram for the model, which give us informations 
on the immune response. Our analysis of the critical behavior is basically supported by the assumption that a finite-size scaling hypothesis can be done for the study of the order parameter as well as the variance and the fourth-order cumulant related to the order parameter. From this analysis we obtain the critical exponents. Our results indicate that the model, although not satisfying detailed balance, is in the same universality class of the Ising model. These results corroborate the "up-down" conjecture of Grinstein et al. [1]. It is interesting to note that, in the present model, at each site a dynamical variable is associated that can attain three values. However, the dynamical rules are invariant under the exchange between two of the values of the dynamical variables. This property brings to the problem a symmetry similar to the one found in the Blume-Emery-Griffiths (BEG) model [21]. In fact, the BEG Hamiltonian is invariant under the exchanging between two of the three values of the spin variable and the line of critical points in this model belongs to the Ising universality class. We also remark that numerical evidences have been found in a variety of models that do not possess detailed balance and fall in the universality class of the Ising model. One example is the majority vote model [22] describing the competition between two Glauber dynamics. Another example is the critical point with Ising-like behavior observed in the Ziff-Gulari-Barshad model [23] with desorption [24].

In the first section we explain the model and their properties. In the two following sections we describe our Monte Carlo simulations for lattices in two and three dimensions as well as the finite-size scaling analysis we performed. Results and discussions are presented in the last section.

\section{The model}

We consider a probabilistic cellular automaton [15] where cells of three types: $T_{H} 0$, $T_{H} 1$ and $T_{H} 2$ occupy the sites of a regular lattice. To each site we attach a dynamical variable $\sigma_{i}$ that takes the values $-1,0$ or +1 as indicated below

$\begin{array}{cccc}\text { cell } & T_{H} 0 & T_{H} 1 & T_{H} 2, \\ \sigma_{i} & 0 & 1 & -1\end{array}$

The dynamical local rules of our model describe the cellular interactions between $T_{H}$ cells and are composed of two processes. One of them describes the transformation of a $T_{H} 0$ cell into either $T_{H} 1$ or $T_{H} 2$ cells whereas the other describes the reversed process. We remark that a $T_{H} 1$ cell never develops into a $T_{H} 2$ cell and vice-versa. The $T_{H} 0$ cell is transformed into a $T_{H} 1\left(T_{H} 2\right)$ cell with probability $p$ if the majority of its nearest neighbors are of the type $T_{H} 1\left(T_{H} 2\right)$ and remains the same with probability $1-p$. When the number of $T_{H} 1$ and $T_{H} 2$ cells in the neighborhood are the same, $T_{H} 0$ cell is transformed into a $T_{H} 1$ (or $T_{H} 2$ ) cell with probability $p / 2$. A $T_{H} 1\left(T_{H} 2\right)$ cell turns into a $T_{H} 0$ cell with probability $r$ independently of the neighbors and remains the same with probability $1-r$. 
The rules so defined are stochastic and of totalistic type [25]. They can be formally expressed by the transition probabilities per site which in this case will be of the form

$$
w_{i}\left(\sigma_{i} \mid \sigma_{i}^{\prime}, S_{i}^{\prime}\right)
$$

with $S_{i}^{\prime}$ defined as

$$
S_{i}^{\prime}=\sum_{\delta} \sigma_{i+\delta},
$$

where the sum is done over the first neighbors of site $i$ in the lattice.

In the first process, in which $\sigma_{i}^{\prime}=0$, transition probability $w_{i}\left(\sigma_{i} \mid 0, S_{i}^{\prime}\right)$ depends on the neighborhood of site $i$ only through the variable $S_{i}^{\prime}$ and is given by

$$
\begin{aligned}
& w_{i}\left(1 \mid 0, S_{i}^{\prime}\right)= \begin{cases}p & \text { if } S_{i}^{\prime}>0, \\
p / 2 & \text { if } S_{i}^{\prime}=0, \\
0 & \text { if } S_{i}^{\prime}<0,\end{cases} \\
& w_{i}\left(0 \mid 0, S_{i}^{\prime}\right)=1-p
\end{aligned}
$$

and

$$
w_{i}\left(-1 \mid 0, S_{i}^{\prime}\right)= \begin{cases}p & \text { if } S_{i}^{\prime}>0, \\ p / 2 & \text { if } S_{i}^{\prime}=0, \\ 0 & \text { if } S_{i}^{\prime}<0 .\end{cases}
$$

In the second process, for which $\sigma_{i}^{\prime}= \pm 1$, the transition probabilities $w_{i}\left(\sigma_{i} \mid 1, S_{i}^{\prime}\right)$ and $w_{i}\left(\sigma_{i} \mid-1, S_{i}^{\prime}\right)$ are defined in terms of $r$ which is related to the mean-life time of cells $T_{H} 1$ and $T_{H} 2$ : a site $i$, at time $\ell$, occupied by a $T_{H} 1\left(T_{H} 2\right)$ cell has the probability $r$ of being occupied by a $T_{H} 0$ cell at time $\ell+1$. They are given by

$$
w_{i}\left(0 \mid+1, S_{i}^{\prime}\right)=w_{i}\left(0 \mid-1, S_{i}^{\prime}\right)=r
$$

and

$$
w_{i}\left(+1 \mid+1, S_{i}^{\prime}\right)=w_{i}\left(-1 \mid-1, S_{i}^{\prime}\right)=1-r .
$$

We remark that

$$
w_{i}\left(-1 \mid+1, S_{i}^{\prime}\right)=w_{i}\left(+1 \mid-1, S_{i}^{\prime}\right)=0 .
$$

These rules have "up-down" symmetry, that is,

$$
w_{i}\left(\sigma_{i} \mid \sigma_{i}^{\prime}, S_{i}^{\prime}\right)=w_{i}\left(-\sigma_{i} \mid-\sigma_{i}^{\prime},-S_{i}^{\prime}\right),
$$

so that we expect, following Grinstein et al. [1] that this probabilistic cellular automaton be in the same universality class as kinetic Ising models.

We remark that the process $T_{H} 0 \rightarrow T_{H} 1$ or $T_{H} 2$ defined by rules (4) and (6) are able to describe the fact that there is a stimulation in the production of $T_{H} 1\left(T_{H} 2\right)$ cells if the majority of cells in the neighborhood of a $T_{H} 0$ cell are of type $T_{H} 1\left(T_{H} 2\right)$. 
Also the fact that a majority of one kind does not allow the production of cells of the other type is comprehended. These are important features of the immune response as is detailed discussed in Refs. [13,14].

\section{Monte Carlo simulations}

We have performed Monte Carlo simulations on hypercubic lattices with periodic boundary conditions. We used lattices with $L^{d}=N$ sites with synchronized update. In one dimension we found no ordered state, as expected from the "up-down" conjecture. Simulations run on square and cubic lattices indicate that the system can present a phase transition from a disordered state to an ordered state. Each simulation starts with a configuration generated at random and average over several simulations were taken to get final results. Each site is updated according to the Markovian rules defined in Eqs. (4)-(9). Then the following procedure is performed: (i) if the site is occupied by a $T_{H} 0$ cell, then a random number is generated and is compared to $p$. If it is less or equal to $p$ then the neighborhood is checked to the presence of $T_{H} 1$ and $T_{H} 2$ cells. If the sum of the dynamical variables of the neighborhood is greater (less) than zero then the site will be occupied by a $T_{H} 1\left(T_{H} 2\right)$ cell in the next time step. If the sum is equal to zero then we generate a random number which is compared to 0.5 to decide if the site will be occupied by a $T_{H} 1$ or $T_{H} 2$ cell. (ii) if the site is occupied by a $T_{H} 1\left(T_{H} 2\right)$ cell, then a random number is generated and if it is less or equal to $r$ then the site will be occupied by a $T_{H} 0$ cell in the next time step. A run is finished when the system reaches the stationary state. The time taken by the system to reach the stationary state depends on the parameters and on the lattice size $L$. Discarding the transient, the data were sampled every $n_{a}$ time units $\left(n_{a}\right.$ reaching 100 Monte Carlo steps when $r$ is close to the critical value $r_{c}$ ). Our simulations were performed fixing $p$ and varying $r$. That is for each $p$ we performed simulations for $r$ varying between 0 and 1 .

The $T_{H} 1$ and $T_{H} 2$ fraction, $x$ and $y$, respectively, as functions of $r$, for a square lattice, are shown in Fig. 1. We can see that there is a critical value $r_{c}$, that for $p=0.3$ is $r_{c}=0.196$, where a phase transition occurs: for $r<r_{c}$ the system is in the ordered phase where $x \neq y$.

We calculated the order parameter

$$
\alpha=\langle|m|\rangle,
$$

where $m=\sum_{i} \sigma_{i} / N$. The average is evaluated numerically as described above. We also compute the "susceptibility", that is the variance

$$
\chi=N\left\{\left\langle m^{2}\right\rangle-\langle|m|\rangle^{2}\right\}
$$

the Binder cumulant [19]

$$
U=1-\frac{\left\langle m^{4}\right\rangle}{3\left\langle m^{2}\right\rangle^{2}}
$$




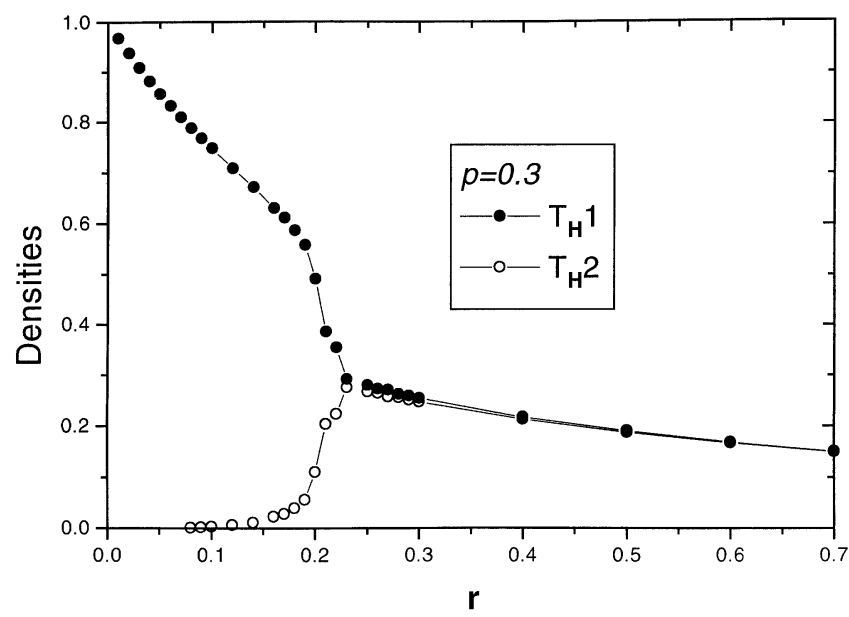

Fig. 1. The $T_{H} 1$ and $T_{H} 2$ fraction, $x$ and $y$, respectively, as functions of $r$, for $p=0.3$. Simulations run on square lattices with $L=30$.

and the surface correlation, introduced in [20],

$$
\tau=\langle\mu(\text { top }) \mu(\text { bottom })\rangle,
$$

where $\mu($ top $)=1(-1)$ if the majority of the sites of the top are in state where $\sigma_{i}=$ $+1(-1)$, and it is zero otherwise. The hypersurfaces designed by top and bottom in previous equation are lines in two-dimensional systems and planes in three-dimensional ones, separated by a distance $L / 2$.

\section{Finite-size scaling}

To find the critical exponents we start from the assumption that a finite-size scaling analysis can be done to study the critical properties of this model. We assume that nonequilibrium continuous phase transitions in this model are characterized by a length scale $\xi$ that diverges, in the infinite system, as $\xi \sim\left|r_{c}-r\right|^{-v}$ where $v$ is the correlation length exponent. Since the (linear) size of the system $L$ scales like the correlation length $\xi$ then any finite system quantity $G_{L}(r)$ will be a homogeneous function of $L$ and $\xi$. From this property it follows that

$$
G_{L}(r)=L^{\theta / v} \widehat{G}\left(\frac{L}{\xi}\right),
$$

or

$$
G_{L}(r)=L^{\theta / v} \widetilde{G}\left(L^{1 / v}\left(r-r_{c}\right)\right) .
$$

In the limit $L \rightarrow \infty$ we have

$$
G_{\infty}(r) \sim\left|r-r_{c}\right|^{-\theta} .
$$




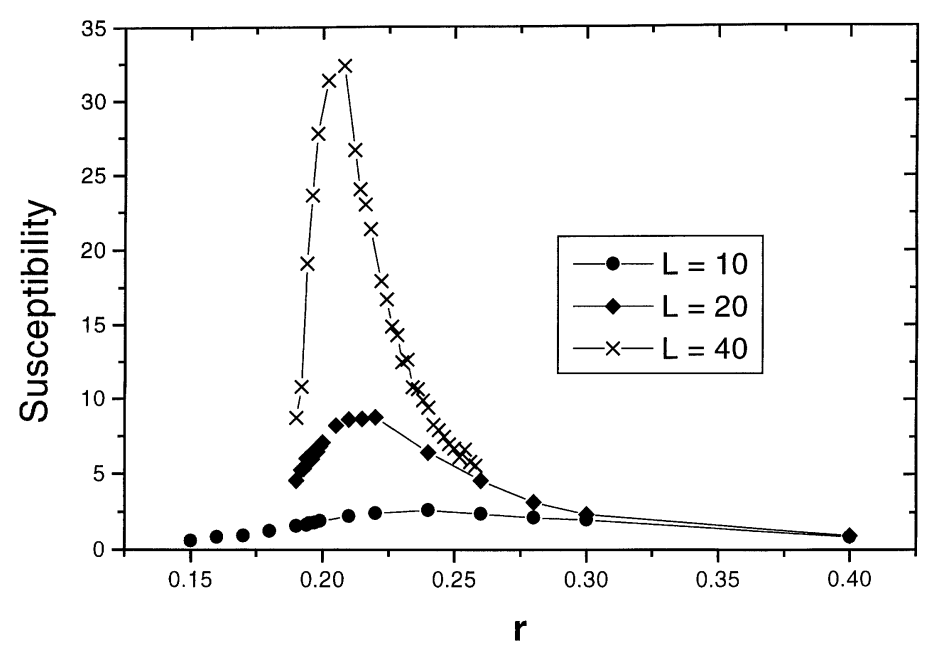

Fig. 2. The susceptibility $\chi_{L}$ as a function of $r(p=0.3)$ for $L=10,20$ and 40 (square lattices).

In the infinite system we expect that near the critical point the order parameter behaves as

$$
\alpha \sim\left|r-r_{c}\right|^{\beta} .
$$

From the above analysis it follows that, for a finite system, the following scaling form for the order parameter should be accomplished:

$$
\alpha_{L}(r)=L^{-\beta / v} f\left(\left(r-r_{c}\right) L^{1 / v}\right),
$$

where $f(x)$ is a universal function.

We calculated the susceptibility for several values of the lattice size $L$ (square lattices) as is shown in Fig. 2. The order of magnitude of the relative error is about $15 \%$ in the critical region and less than $5 \%$ out of that region. We can see that the susceptibility has a peak for a value of $r$ (fixed $p$ ). As $L$ is increased this peak turns to be sharper and we assume that for the infinite system $\chi$ diverges at the critical point as

$$
\chi \sim\left|r-r_{c}\right|^{-\gamma} .
$$

We also assume a finite-size scaling form for $\chi$ as

$$
\chi_{L}(r)=L^{\gamma / v} g\left(\left(r-r_{c}\right) L^{1 / v}\right),
$$

where $g(x)$ is a universal function.

The fourth-order cumulant obeys the following scaling relation

$$
U_{L}(r)=h\left(L^{1 / v}\left(r-r_{c}\right)\right),
$$

where $h(x)$ is a universal function. When $r \rightarrow r_{c}$ then $U_{L}\left(r \rightarrow r_{c}\right)=U^{*}$ and it follows that $U^{*}$ is universal. That is in the critical point $U$ attains a universal value that does 


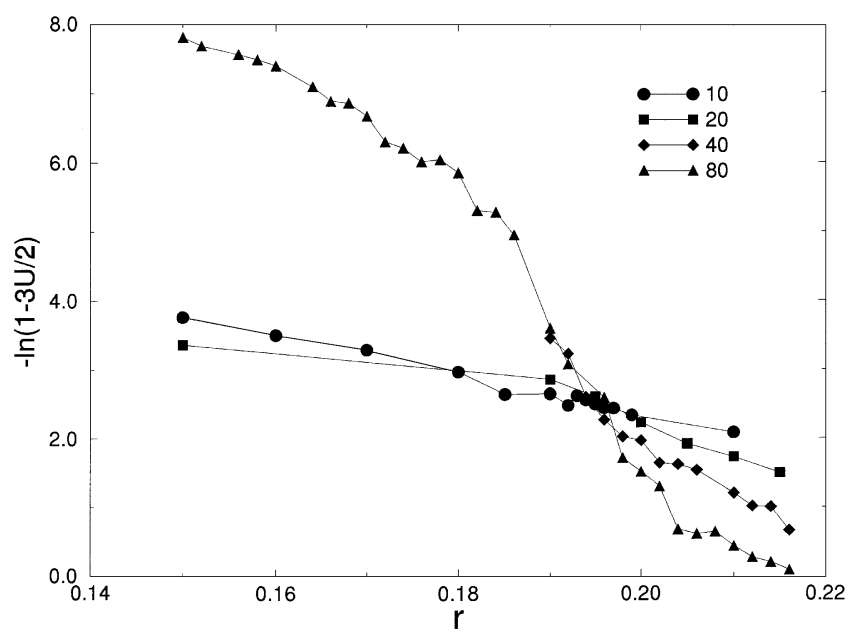

Fig. 3. Binder cumulant $U_{L}$ versus $r(p=0.3)$ for square lattices with $L=10,20,40$ and 80 .

not depend on the lattice size. We used the cumulant method [19], which is based on this fact, to determine the critical values $r_{c}(p)$. For Ising models on the square lattice with periodic boundary conditions, it has been found [26] that $U^{*} \simeq 0.61$.

The surface correlation $\tau$, defined in Eq. (14), was used as another way of determining the critical parameter $r_{c}(p)$. This quantity, which scales as $L^{0}$, was proposed $[20,27]$ in the context of equilibrium phase transitions as a new type of finite-size scaling algorithm. Later, it has been shown to be useful even to study far from equilibrium phenomena [28]. From the analysis of $\tau$ it is possible to verify if the system is in a single-domain state $(\tau \rightarrow 1)$ or is not correlated $(\tau \rightarrow 0)$ [29].

In what follows we report the finite-size scaling analysis based on our Monte Carlo simulations. In Fig. 3 we show $U_{L}$ as a function of $r$ for a fixed value of $p$ and for different lattice size $L$. All curves intercept in the critical point $r_{c}(p)$. From these data we construct the phase diagram, for the square lattice, shown in Fig. 4. The points in the critical line are such that $U^{*}=0.607 \pm 0.005$, except for the point $(p, r)=(1,1)$ where the cumulant results indicate that the transition is not continuous. Fig. 5 shows the search for the critical parameter $r_{c}(p)$ using the surface correlation. The result is in complete agreement with that obtained by the cumulant method.

From the scaling relation (19) we derive the following expression for $r=r_{c}$ :

$$
\ln \alpha_{L}\left(r_{c}\right)=A-\frac{\beta}{v} \ln L
$$

where $A$ is a constant. To evaluate the ratio of the critical exponents $\beta / v$ we plot $\ln \alpha_{L}\left(r_{c}\right)$ with $r_{c}$ obtained from the cumulant results versus $\ln L$ as is shown in Fig. 6 . We did this procedure for $0<p<1$ and estimated this ratio as $\beta / v=0.126 \pm 0.014$.

From the scaling relation (21) we derive the following relation $r=r_{c}$ :

$$
\ln \chi_{L}\left(r_{c}\right)=B+\frac{\gamma}{v} \ln L
$$




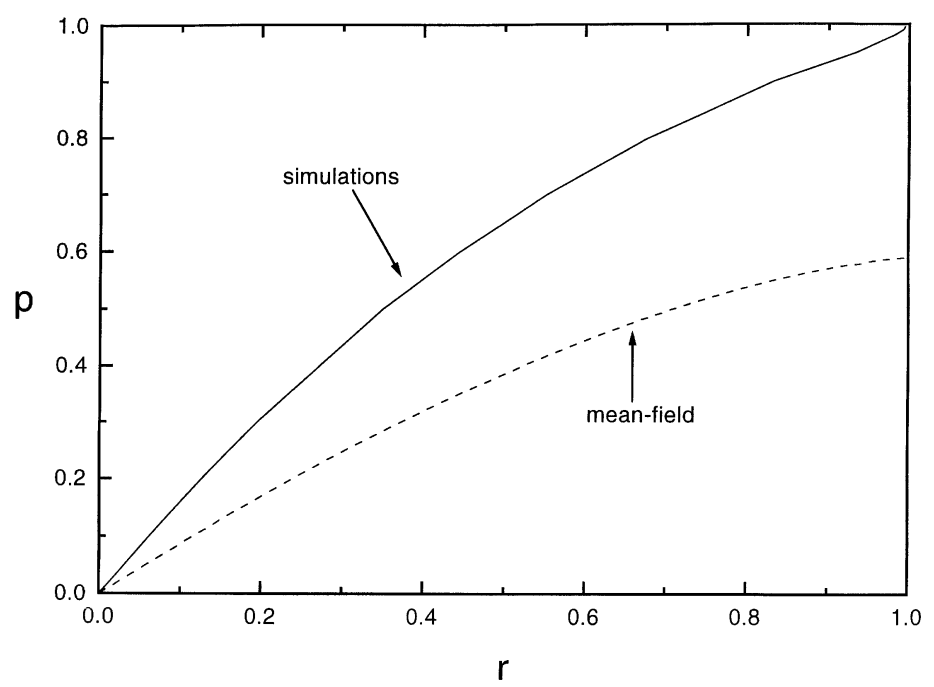

Fig. 4. The phase diagram in the $r-p$ plane for square lattices. Critical points were obtained by the cumulant method (continuous line). For the sake of comparison we have included results from pair mean-field approximation of Ref. [15] (dashed line).

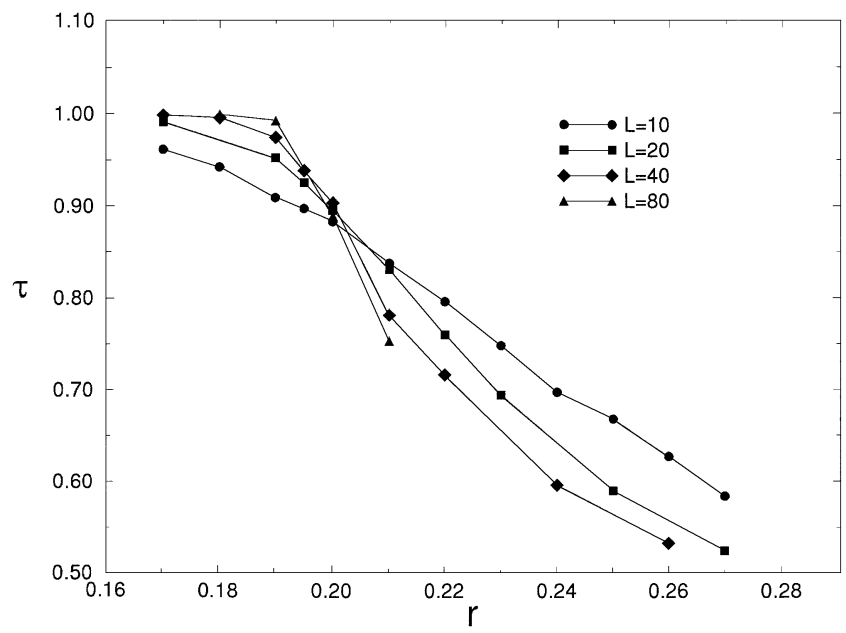

Fig. 5. Surface correlation as a function of $r$ for $p=0.3$ and $L=10,20,40$ and 80 (square lattices). The critical value is $r_{c}=0.198 \pm 0.002$.

where $B$ is a constant and $r_{c}$ is obtained from the cumulant method. The ratio $\gamma / v$ is obtained by plotting $\ln \chi_{L}\left(r_{c}\right)$ as a function of $\ln L$ as is shown in Fig. 7. We evaluated $\gamma / v$ for several values of the parameters and our result is $\gamma / v=1.70 \pm 0.05$. Finally, we quote the results obtained for three-dimensional lattices. In Fig. 8 we show the 


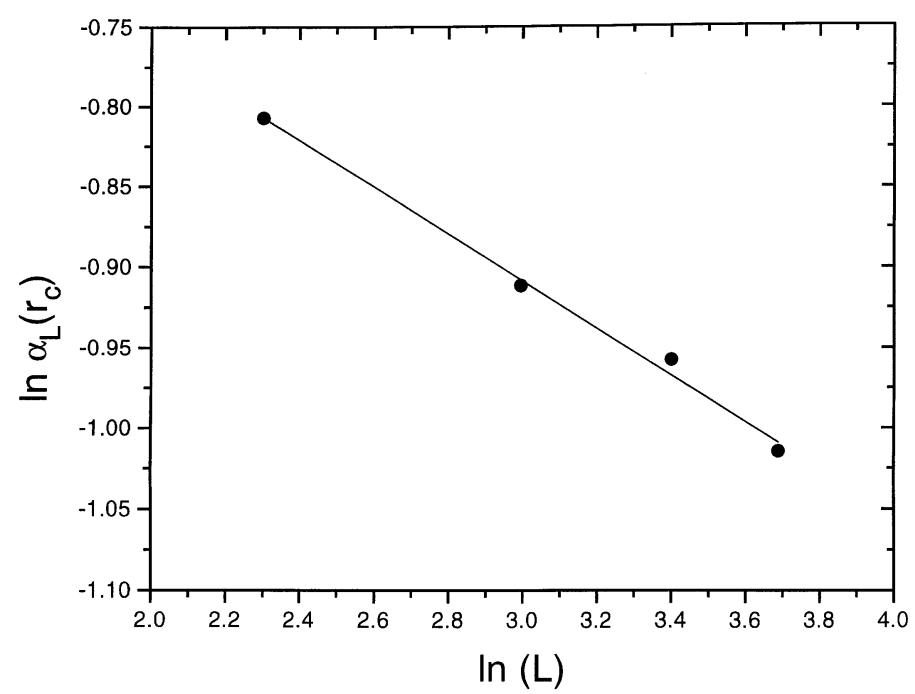

Fig. 6. $\log -\log$ plot of $\alpha_{L}\left(r_{c}\right)$, with $r_{c}$ obtained from the cumulant results, versus $L$. The slopes of this line gives the ratio $\beta / v$. We did the same procedure for $0<p<1$ and estimated this ratio as $\beta / v=0.126 \pm 0.014$ (square lattices).

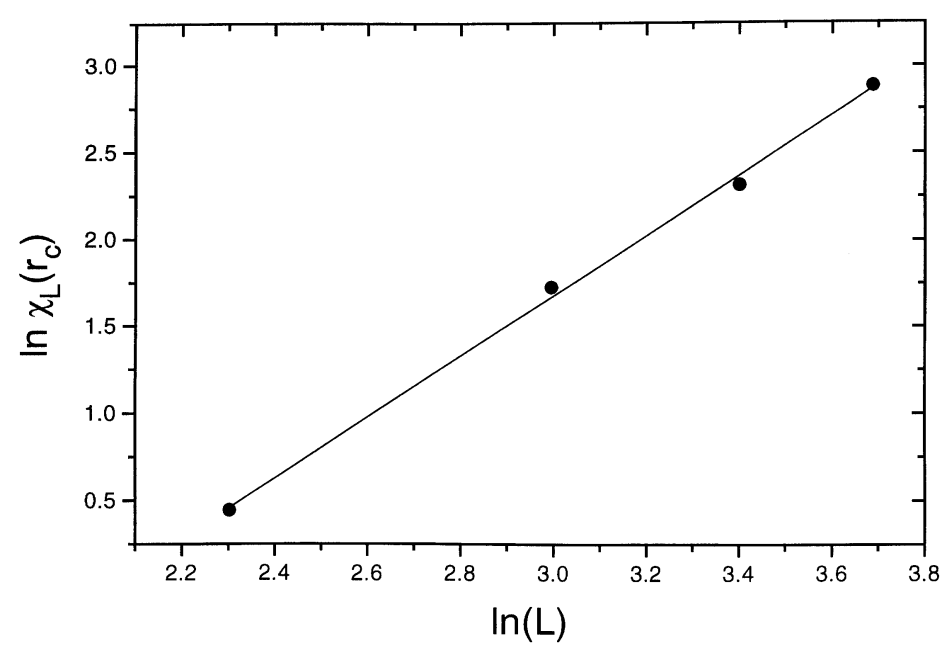

Fig. 7. Log-log plot of $\chi_{L}\left(r_{c}\right)$ versus $L$. The ratio $\gamma / v$ is obtained by the slope of the line. We did the same procedure for $0<p<1$ and estimated the ratio $\gamma / v=1.70 \pm 0.05$ (square lattices).

plot of the fourth-order cumulant against $r$, for $p=0.3$. We found $r_{c}=0.545 \pm 0.003$ using cubic lattices with $L=10,15$ and 20. Preliminary estimates suggest the values $0.59 \pm 0.05$ for the ratio $\beta / v$ and $1.92 \pm 0.1$ for $\gamma / v$ (to be compared with 0.52 and 1.98 for the three-dimensional Ising model [30]). 


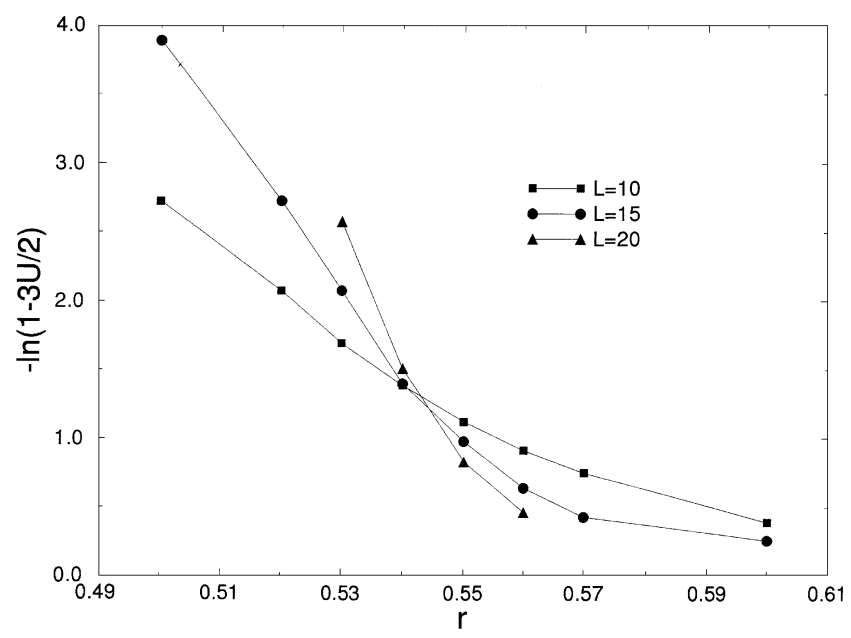

Fig. 8. Binder cumulant $U_{L}$ versus $r(p=0.3)$ for cubic lattices with $L=10,15$ and 20 .

\section{Conclusions}

We have simulated numerically a probabilistic cellular automaton representing the dynamics of three types of biological cells, $T_{H} 0, T_{H} 1$ and $T_{H} 2$. The dynamical rules are invariant under the exchange of $T_{H} 1$ and $T_{H} 2$ cells resulting in an "up-down" symmetry. The automaton displays one disordered state with the same symmetry which is spontaneously broken, as one varies the parameters, giving rise to a nonequilibrium phase transition and an ordered state with the predominance of either $T_{H} 1$ or $T_{H} 2$.

The numerical results obtained in the square and cubic lattices show that the disordered state is stable, at fixed $p$, for high values of the death probability $r$. As we decrease $r$ a symmetry breaking takes place at a critical value $r_{c}$ below which the system is in the ordered phase. We observe that, for fixed $p$, as $r$ is decreased the model always presents a kinetic phase transition. The phase diagram shown in Fig. 4, obtained here from numerical simulations (on square lattices) is qualitatively different from the one obtained in Ref. [15], where mean-field approaches have been used. Mean-field results indicated that the ordered state could be stable for any value of $r$ for high values of $p$ whereas the present results show that, given any value of $p<1$, there is always a value $r_{c}<1$ above which the system is in the disordered state. We believe that the present numerical results are in agreement with immunological responses, since the ordered state (immune response differentiated) should not be stable for any value of $r$ even if $p$ is large. This assertion is based on the following rationale: high values of $r$ correspond to situations where the organism is not exposed to a high level of infection and in this case it should not give a differentiated immune response, characterized by the polarization of $T_{H}$ cells. This phenomenon is experimentally verified in strains of mice normally resistant to a high-level infection of Trichura muris that become susceptible to a low-level infection [14]. 
The critical exponents were evaluated from numerical simulations on lattices, of sizes ranging from $L=10$ to $L=80$, in two dimensions and with the help of finite-size scaling analysis. They are, consistent with Ising-like behavior. The same behavior is observed along all the critical line, except at the point $(r, p)=(1,1)$ where the transition seems to be discontinuous. Preliminary results for the three-dimensional lattice reinforce the hypothesis of universality between this model and Ising one. These results are consistent with the conjecture [1] for which automata whose dynamic rules possess "up-down" symmetry and respect the symmetry of the lattice should belong to the Ising universality class.

\section{References}

[1] G. Grinstein, C. Jayaprakash, H. Yu, Phys. Rev. Lett. 55 (1985) 2527.

[2] P. Grassberger, Z. Phys. B 47 (1982) 365.

[3] H.K. Janssen, Z. Phys. B 42 (1981) 151.

[4] R. Dickman, Int. J. Mod. Phys. C 4 (1993) 271.

[5] J.E. Satulovsky, T. Tomé, Phys. Rev. E 49 (1994) 5073.

[6] H. Matsuda, N. Ogita, A. Sasaki, K. Sato, Prog. Theoret. Phys. 6 (1992) 1035.

[7] P. Grassberger, Math. Biosci. 63 (1983) 157.

[8] N. Boccara, K. Cheong, M. Oram, J. Phys. A 27 (1994) 1585.

[9] S.A. Kauffman, J. Theoret. Biol. 22 (1969) 437.

[10] G. Weisbuch, H. Atlan, J. Phys. A 21 (1988) L189.

[11] F. Celada, P.E. Seiden, Immunol. Today 13 (1992) 56.

[12] D. Stauffer, G. Weisbuch, Physica A 180 (1992) 42.

[13] A. Brass, R.K. Grencis, K.J. Else, J. Theoret. Biol. 166 (1994) 189.

[14] A. Brass, A.J. Bancroft, M.E. Clamp, R.K. Grencis, K.J. Else, Phys. Rev. E 50 (1994) 1589.

[15] T. Tomé, J.R. Drugowich de Felício, Phys. Rev. E 53 (1996) 3976.

[16] T.R. Mosmann, R.L. Coffman, Adv. Immunol. 46 (1988) 111.

[17] K.J. Else, R.K. Grencis, Immunol. 72 (1991) 508.

[18] P. Scott, H.E. Kaufmann, Immunol. Today 12 (1991) 346.

[19] K. Binder, Applications of the Monte Carlo Method in Statistical Physics, Springer, Berlin, 1984.

[20] P.M.C. de Oliveira, Europhys. Lett. 20 (1992) 621.

[21] M. Blume, V.J. Emery, R.B. Griffiths, Phys. Rev. A 14 (1971) 1071.

[22] M.J. de Oliveira, J. Stat. Phys. 66 (1992) 273.

[23] R.M. Ziff, E. Gulari, I. Barshad, Phys. Rev. Lett. 56 (1986) 2553.

[24] T. Tomé, R. Dickman, Phys. Rev. E 47 (1993) 948.

[25] S. Wolfram, Rev. Mod. Phys. 55 (1983) 601.

[26] D.P. Landau, in: V. Privman (Ed.), Finite Size Scaling and Numerical Simulation of Statistical System, World Scientific, Singapore, 1990, p. 223.

[27] S. Moss de Oliveira, P.M.C. de Oliveira, F.C. de Sá Barreto, J. Stat. Phys. 78 (1995) 1619.

[28] M. Silvério Soares, J. Khamphorst Leal da Silva, F.C. Sá Barreto, Phys. Rev. B 55 (1997) 1021.

[29] P.M.C. de Oliveira, S.M. Moss de Oliveira, C.E. Cordeiro, D. Stauffer, J. Stat. Phys. 80 (1995) 1433.

[30] J.M. Yeomans, Statistical Mechanics of Phase Transitions, Claredon Press, Oxford, 1992. 